\title{
Performance Analysis of Switched Control Systems Under Common-source Digital Upsets Modeled by MDHMM
}

\author{
Rui Wang $\left(\mathbb{D},{ }^{1}\right.$ Yanxiao Li, ${ }^{2}$ Hui Sun $\left(\mathbb{D},{ }^{1,3}\right.$ Youmin Zhang, ${ }^{4}$ and Yigang Sun $\mathbb{D}^{5}$ \\ ${ }^{1}$ Information Engineering and Automation College, Civil Aviation University of China, Tianjin, China \\ ${ }^{2}$ Technology Innovation Center, China Electronics Technology Avionics Co., Ltd., Chengdu, China \\ ${ }^{3}$ Department of Engineering Design and Mathematics, University of the West of England, Frenchy Campus, Coldharbour Lane, \\ Bristol, BS 161QY, UK \\ ${ }^{4}$ Department of Mechanical, Industrial and Aerospace Engineering, Concordia University, Montreal, Canada \\ ${ }^{5}$ College of Aeronautical Engineering, Civil Aviation University of China, Tianjin, China
}

Correspondence should be addressed to Rui Wang; ruiwang@cauc.edu.cn and Hui Sun; h-sun@cauc.edu.cn

Received 21 July 2018; Accepted 18 October 2018; Published 5 November 2018

Guest Editor: Junpei Zhong

Copyright ( 2018 Rui Wang et al. This is an open access article distributed under the Creative Commons Attribution License, which permits unrestricted use, distribution, and reproduction in any medium, provided the original work is properly cited.

\begin{abstract}
This paper proposes the theoretical model to analyze the performance degradation of control systems subject to common-source digital upsets. In this paper, a multidimensional hidden Markov model (MDHMM) is used to characterize the correlated upsets and reveals the relationship between complex environments and stochastic random digital upsets injected into the control systems. These digital upsets coming from artificial complex environments are operated on distributed redundant processing controllers. Furthermore, this paper develops the theoretical analysis model for performance degradation of control systems under commonsource digital interferences modeled by MDHMM. Theoretical estimations for different redundant configurations are analyzed. Then corresponding simulation verifications for a specific control system are also conducted in details and compared with the theoretical analysis results. These analyses can help to select an optimal redundant design and provide an example for control systems design. This analysis also helps to balance the performance of system, reliability of system, and costs of system design in applications.
\end{abstract}

\section{Introduction}

Modern aircraft uses more digital fly-by-wire control systems benefited from new advancements in electronics, computer, and control technologies, and the fly-by-wire flight control system is playing more important role for the aircraft safety, stability and maneuverability. However, it is inevitable to encounter the challenging threats from the natural and manmade electromagnetic interferences such as lightning, high intensity radiated fields (HIRF). Especially, HIRF is one of the main threat sources for civil digital flight control systems. These interferences radiation come from radars, radios, or other transmitters applied from ground, in the air, or at the sea. During flight, the electromagnetic environments can interrupt the controller and generate so called "digital upsets" which will reverse the binary bits in controllers from digit ' 0 ' to ' 1 ' or from digit ' 1 ' to ' 0 '. Even though some controllers possess the recoverable capability or these errors may not induce the functional failures immediately, the accumulation of the errors will cause a large deviation from the operation references, affects the stability of the flight control system performance, even leads to the fatal failure. This is the serious potential threat for the loss of control and the whole aircraft control system safety. Therefore, it is necessary to establish a model to analyze and monitor the performance degradation of flight control systems affected by correlated digital upsets under electromagnetic environments. The focus on monitoring stability of civil flight control systems meets the requirements of flight safety concern and can provide a model verification method for civil aircraft airworthiness certification and designs.

The so named digital upset process has the binary random variables, 0 and 1 . For example, if there is an upset injected into flight controllers, the variable takes on ' 1 ' or ' 0 ' otherwise. The current literatures indicate that digital upsets can be characterized by Markovian models 
originally proposed in [1]. The performance analysis methods of flight control systems were carried out in some literatures when considering Markovian upset processes [2-6]. Reference [2] proposed a disturbance model represented by Markovian upset processes and developed the performance stability analysis theory and verification method. Reference [3] discussed the necessary and sufficient conditions of the mean-square stable performance analysis as a result of electromagnetic interferences. Reference [4] developed a stochastic electromagnetic disturbance model injected into the closed-loop jump linear system. Reference $[5,6]$ proposed a stochastic hybrid model used to describe neutron-induced single event upset processes and analyze the corresponding performance of recoverable digital Boeing 737 flight control system under this kind of environments. The simulated neutron experiments were also conducted at the NASA Langley Research Center. However, physical experiments in $[7,8]$ discovered that the stochastic digital upsets cannot be characterized by general order Markovian models precisely. Reference [8-11] developed a general model for analyzing the performance degradations of flight control systems subject to independent upset processes induced by HIRF environments and presented a burst model by adding more upsets in order to extend the duration of upset processes. Therefore, this method can only provide the upper bound for performance degradation analysis under independent upsets. In reality, the electronic devices are located at a limited electronic equipment compartment of the aircraft; then they are exposed in a common-source interference area with a high probability. Therefore, Reference [12, 13] proposed a more accurate model to describe the relationship between the environment interference and independent digital upsets injected into flight control systems by using the hidden Markov model (HMM). Furthermore, performance analysis method based on HMM model of the switched linear systems are considered and proposed as well. All of the above researches considered the independent digital upsets injected into the flight control system and, however, ignored common-mode characteristics of the upsets. Reference [14] presented a method to describe common-source interferences by using the multidimensional hidden Markov model (MDHMM).

MDHMM, a signal statistical analysis model, is widely used in the fields of pattern recognition and financial areas, and many researches focus on the applications of movement segments for controlling a multifunctional prosthetic hand, measurement assessment and face recognition, etc. [15-18]. Reference [19] proposed a zero-delay MDHMM in terms of the fitting capacity and prediction power to capture the evolution of the foreign exchange rate data under different frequent trading environments. Furthermore, this method demonstrates that MDHMM can better and more accurately characterize the dynamics than HMM does. However, MDHMM has not been applied in revealing the hidden characteristics of the upsets coming from the commonsource environments yet. The initial value selection strategy is also a focus of the relevant research. In literature [20], the $k$-means algorithm is used to estimate the initial observation probability matrix, while the initial stationary probability vector and the initial state transition probability matrix are uniformly initialized. Recently, with the development of artificial intelligence, some algorithms such as particle swarm, simulated annealing and ant colony are used to carry out parameter training and have achieved good results. However, such algorithms also have huge computational burden.

Therefore, the motivation of this paper is to develop a theoretical model and the simulation verification method to analyze the performance degradation affected by commonsource digital upsets modeled by MDHMM.

The two main contributions are addressed in this paper. First, this paper uses MDHMM to reveal the inherent physical causes of common-source digital interferences caused by environment to multichannel flight control systems. In the current literatures, relevant scholars gradually established the accurate interferences characteristics of single-channel flight control systems in HIRF environment through Bernoulli distribution, Markov process and hidden Markov process. For a multichannel flight control system, the digital interference characteristics of each control channel are the same as the single-channel flight control system. However, the interferences of different control channels has a common-source characteristic. Under the authors' knowledge, the authors have not found a suitable method to describe the digital random interferences characteristics of multichannel flight control systems. So far literatures only describe the methods using the independent stochastic process to describe the interferences of each channel; however, this method cannot describe the common-source characteristic. Therefore, the theoretical calculation differs greatly from the actual data. Second, the theoretical analysis model of flight control system performance degradation is developed which integrates the system dynamics the aircraft undertakes, the redundant structure the flight design characterizes, and the MDHMM model the disturbances satisfy. This innovative provides a theoretical basis for the design of the redundancy structure of the flight control system. It is known that the commonsource interferences on each channel are not independent of each other. Through theoretical analysis and simulation verifications, this paper observes that the reliability improvement effect gradually decreases by increasing the number of control channels in the HIRF environment. This paper is the consecutive research in [14] and will develop the theoretical performance degradation model by using MDHMM.

The arrangement of the paper is addressed as follows. Section 2 describes the general performance modeling of redundant flight control systems subject to common-source digital upsets by using MDHMM. Theoretical performance degradation analysis method of the redundant flight control systems is presented by applying MDHMM to characterize the correlated digital upsets in Section 3. An example applied in Boeing 747 flight control system and the simulation verification analyses are shown in Sections 4 and 5, respectively. The last section summarizes the main results.

\section{Modeling of Redundant Flight Control Systems Subject to Correlated Digital Upsets}

2.1. Performance Model of the Redundant Flight Control Systems Subject to the Disturbance Model. The distributed 


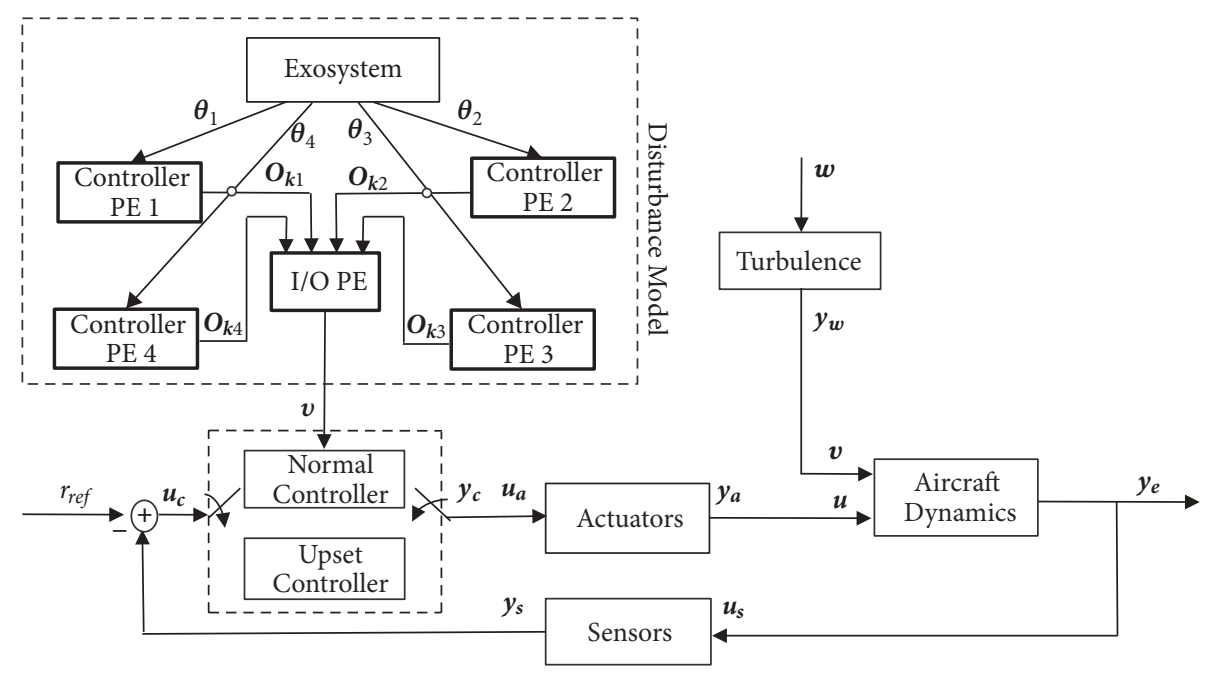

FIGURE 1: Scheme of flight control system subject to digital upsets.

redundant flight control system with disturbance model is described in Figure 1. The disturbance model includes the exosystem and the distributed redundant platform. The exosystem is used to generate common-source correlated digital upsets fed to distributed redundancy platform. This platform is composed of controller processing element units (CPE units); furthermore, this platform is the copy of the redundant controller-configuration of flight systems. The platform output is used to characterize the effect of stochastic digital upsets applied on the platform. CPE units can calculate updates of the control law at each sample time and can be recoverable. The logic of I/O PE is 'AND', and the platform output drives the switched linear control system [8]. Therefore, system performance degradation can be studied via the platform when subject to such digital upsets. Since digital I/O PE output, $v(k)$, works as a switched binary signal, it has two states, ' 0 ' and ' 1 '. ' 0 ' represents the normal state without interference. ' 1 ' represents the upset state which indicates that there is an interference applied on control systems. Then the flight control system switches between the normal and the upset mode. Some other assumptions are the same with those in [8] in details.

In this paper, a discrete linearized flight control system driven by the switching signal, $v(k)$, is described below:

$$
\begin{gathered}
\boldsymbol{x}(k+1)=\boldsymbol{A}_{v(k)} x(k)+\boldsymbol{B}_{v(k)} \boldsymbol{w}(k)+\boldsymbol{r}_{r e f}(k) \\
\boldsymbol{y}(k)=\boldsymbol{C}_{v(k)} \boldsymbol{x}(k)
\end{gathered}
$$

The binary switching signal $v(k)$ reflects whether the flight control system is interfered by digital upsets at time $k$. That is, when $v(k)=0$, flight control system operates in the nominal mode characterized by state space model $\left(\boldsymbol{A}_{0}, \boldsymbol{B}_{0}, \boldsymbol{C}_{0}\right)$, otherwise, the system operates in the upset mode represented by $\left(\boldsymbol{A}_{1}, \boldsymbol{B}_{1}, \boldsymbol{C}_{1}\right)$. Furthermore, $\boldsymbol{w}(k)$ is the white Gaussian noise input. $\boldsymbol{r}_{r e f}(k)$ is the reference signal. The linear switched flight control system uses Boeing 747 linearization models derived in details in [8]. The closed-loop control system works as the nominal mode, and the open-loop control system is the upset mode.

The error system as shown in Figure 2 is used to analyze the performance degradation of the flight control system subject to digital stochastic upsets driven by the switching signal, $v(k)$. In the error system, there are two copies of system (1a) and (1b) with the same input, $\boldsymbol{w}(k)$. One system acts as a reference system and works in nominal mode continuously. Another upset system is switched by the switching signal, $v(k)$. Then the difference of the corresponding outputs generates the error signal, $y_{e}(k)$. Then, a new error system dynamics is described by the following switched linear system:

$$
\begin{aligned}
\boldsymbol{x}_{e}(k+1) & =\boldsymbol{A}_{e, v(k)} x_{e}(k)+\boldsymbol{B}_{e, v(k)} \boldsymbol{w}(k) \\
\boldsymbol{y}_{e}(k) & =\boldsymbol{C}_{e, v(k)} \boldsymbol{x}_{e}(k)
\end{aligned}
$$

where $\boldsymbol{A}_{e, v(k)}=\operatorname{diag}\left(\boldsymbol{A}_{v(k)}, \boldsymbol{A}_{0}\right), \boldsymbol{B}_{e, \boldsymbol{v}(k)}=\left[\boldsymbol{B}_{\boldsymbol{v}(k)}^{T}, \boldsymbol{B}_{0}^{T}\right]^{T}$ and $\boldsymbol{C}_{e, \boldsymbol{v}(k)}=\left[\boldsymbol{C}_{\boldsymbol{v}(k)},-\boldsymbol{C}_{0}\right] \cdot \boldsymbol{x}_{e}(k)=\left[\boldsymbol{x}_{u}^{T}(k) \boldsymbol{x}_{r}^{T}(k)\right]^{T}$ is the state vector of the error system. Then the index, $\boldsymbol{J}_{w, e}$, is the mean power and used to measure the degradation of the performance. $\boldsymbol{J}_{w, e}$ is a function of the correlated upset processes, $\boldsymbol{\theta}_{i}$, and the structure of the redundant design.

$$
J_{w, e}=\lim _{k \rightarrow \infty} \boldsymbol{E}\left\{\left\|y_{e}(k)\right\|^{2}\right\}
$$

The error system is mean-square stable (MSS) when the limit exists. This is also consistent with the definition in [8].

2.2. Correlated Digital Upsets Processes Modeled by MultiDimensional Hidden Markov Model (MDHMM). As proposed in [14], MDHMM model can better describe the correlated common-source characteristics among the hidden environments and observed digital upsets. The digital upsets are two-state stochastic processes with states strong and weak. In general, the observed digital upsets processes can be modeled by the first-order Markov process. However, 


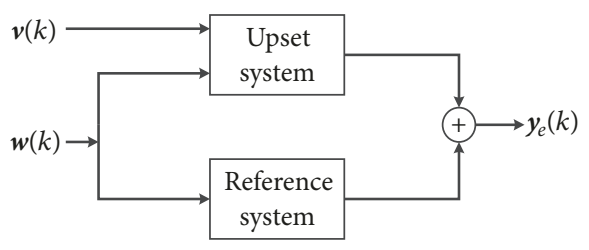

FIGURE 2: Switched error system structure for flight performance.

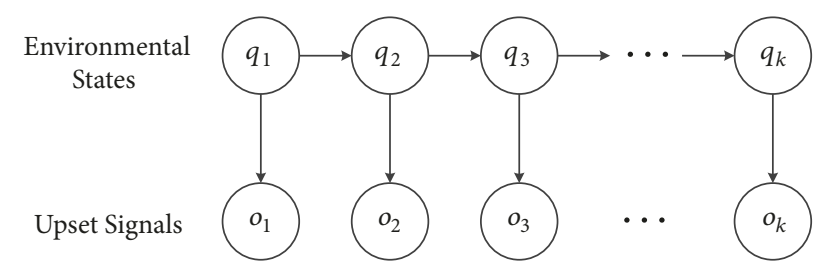

Figure 3: Hidden Markov model structure represented by Bayesian network.

in reality, the interrupt processes from the electromagnetic environment are correlated instead of independent. Further, the common-source upsets affect the different units with the different probabilities. The different interference levels are determined by the locations of the units, such as different angles between the electromagnetic interference and the $\mathrm{CPE}$ units.

As defined in [14] and shown in Figure 3, some parameter definitions are shown below. Supposing the MDHMM model is defined as $\boldsymbol{\lambda}=\left(\boldsymbol{S}, \boldsymbol{V}, \boldsymbol{A}_{h}, \boldsymbol{B}_{h}^{f}, \boldsymbol{\pi}\right), f \in\{1,2, \ldots, F\}$, then the corresponding parameter definitions are placed below.

(1) $\left.\boldsymbol{S}=\left\{S_{1}, S_{2}, \ldots, S_{N}\right\}\right)$ is a N-states set.

(2) $V^{f}=\left\{V_{1}^{f}, V_{2}^{f}, \ldots, V_{M_{f}}^{f}\right\}$ is the observation set with $f$-dimension. $M_{f}$ is the observation number, $o_{k}^{f}$ is the $f$-th output observation variable of MDHMM at time $k, \mathrm{o}_{k}^{f} \in \boldsymbol{V}^{f}$. The output observation vector is $o_{k}=\left(o_{k}^{1}, o_{k}^{2}, \ldots, o_{k}^{F}\right)$.

(3) $A_{f}=\left[a_{i j}\right]$ is state transition matrix for the hidden Markov process, $a_{i j}=P\left\{q_{k}=S_{j} \mid q_{k-1}=S_{i}\right\}, i, j \in$ $\{1,2, \ldots, N\}, f \geq 1 . q_{k}$ is the hidden Markovian state at time $k, q_{k} \in \boldsymbol{S}$.

(4) $\boldsymbol{B}_{h}^{f}=\left[b_{j}^{f}(l)\right]$ is an $N \times \mathrm{M}_{f}$ observation probability matrix, $b_{j}^{f}(l)=P\left\{o_{k}^{f}=V_{l}^{f} \mid q_{k}=S_{j}\right\}, \quad j \in\{1,2, \ldots, N\}, l \in$ $\left\{1,2, \ldots, M_{f}\right\}, f \in\{1,2, \ldots, F\}, k \geq 1$.

(5) $\pi=\left(\pi_{1}, \pi_{2}, \ldots, \pi_{N}\right)$ is the initial stationary probability distribution, where $\pi_{i}=P\left\{q_{0}=S_{i}\right\}, i \in\{1,2, \ldots, N\}$.

In fact, in MDHMM model, the hidden states imply the real electromagnetic states belonging to $S$ with two states, that means $N=2$. Suppose that the state transition of the hidden process satisfies the Markov characteristics. In this paper, all units are subject to the environments. There are $F$ redundant control units. Therefore, the hidden-process transition matrix $\boldsymbol{A}_{f}$ and each corresponding observation matrix $\boldsymbol{B}_{h}^{f}$ are all $2 \times 2$.

Take the three-CPEs configuration as an example and all CPEs are subject to simulated common-source digital upsets processes with 4000 samples and $174 \mathrm{~Hz}$ sampling frequency.
Suppose the initial condition of the flight control systems are placed in normal modes. MDHMM is applied to train the original common-source signals, and the corresponding environmental and observation matrices are estimated and shown below:

$$
\begin{aligned}
A_{h} & =\left(\begin{array}{ll}
0.9990326 & 0.0009674 \\
0.0322089 & 0.9677911
\end{array}\right) \\
B_{h}^{1} & =\left(\begin{array}{ll}
0.9993127 & 0.0006873 \\
0.1779482 & 0.8220518
\end{array}\right) \\
B_{h}^{2} & =\left(\begin{array}{ll}
0.9993167 & 0.0006833 \\
0.1777482 & 0.8222518
\end{array}\right) \\
B_{h}^{3} & =\left(\begin{array}{ll}
0.9993157 & 0.0006843 \\
0.1779382 & 0.8220618
\end{array}\right)
\end{aligned}
$$

The enlarged part of Figures 4(d)-4(f) can more clearly show the common-source characteristics of the digital upset modeled by MDHMM model over the mentioned configuration. Compared to the existing HMM model proposed in [10], MDHMM can be better used to model the commonsource digital interferences. Specially, MDHMM model can be changed into HMM model when $F=1$.

In order to analyze the multi-channel digital upsets by MDHMM, first, it is necessary to estimate the model parameters. Unfortunately, the optimal estimate of the model parameters cannot be obtained by finite length of the observed sequence [21]. However, the local optimal estimate satisfies the specified precision by numerical algorithm and the most classical parameter training algorithm is Baum-Welch algorithm. However, the initial state transition probability matrix $\boldsymbol{A}_{h_{0}}$ and initial observation probability matrix $\boldsymbol{B}_{h_{0}}^{f}$ should be specified. Furthermore, the algorithm for nonconvex function is locally convergent, therefore, the final results are very sensitive to the initial value selection. If the initial value selection is not appropriate, the algorithm can only get to the local optimal solution or is unable to converge. While the appropriate initial value selection not only make the local optimal solution close to the global optimal solution, but also accelerate the process of the algorithm.

In order to meet the real-time requirements in practical applications, fast parameter training methods must be considered for the initial value selection strategy. The proper initial value selection strategy can greatly accelerate the training process of the model. However, the strategy of parameter initialization is not fixed. For example, according to the practical flight program, the initial stationary probability vector takes the value of $\left\{\pi_{0}=1, \pi_{1}=0\right\}$, and does not need to be estimated since flight systems start up normally. Due to these characteristics, a priori knowledge for the initialization of model parameters can be obtained, and the accuracy of initialization is also increased.

It is stated that the hidden process is a first-order Markov process in MDHMM. Therefore, if every observation probability matrix is a unit matrix, then the digital upsets in each controller is also a first-order Markov process. However, in 


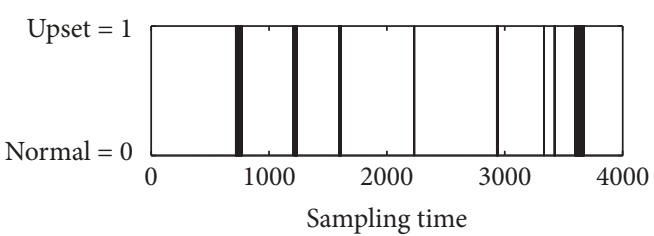

(a) Global upsets in Channel 1

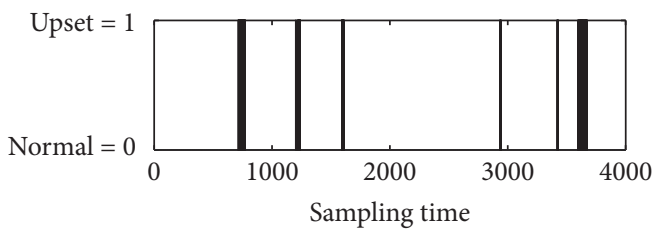

(b) Global upsets in Channel 2

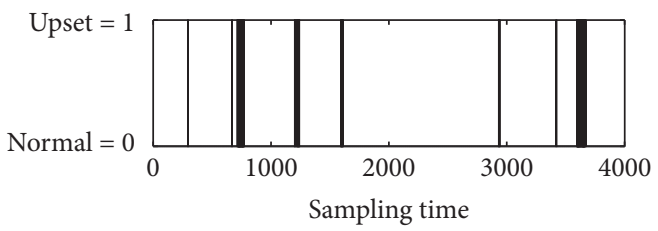

(c) Global upsets in Channel 3

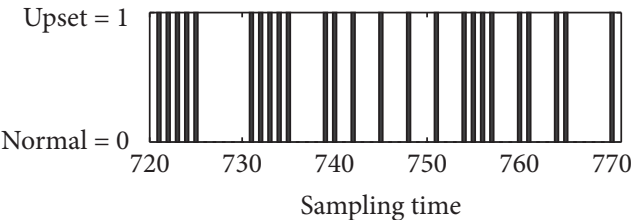

(d) Detailed upsets in Channel 1

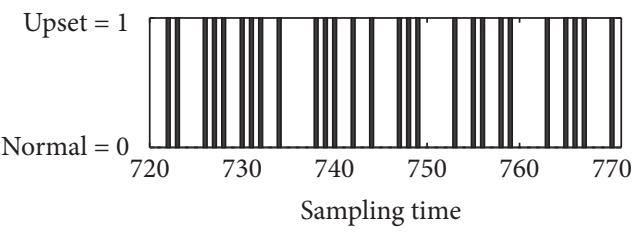

(e) Detailed upsets in Channel 2

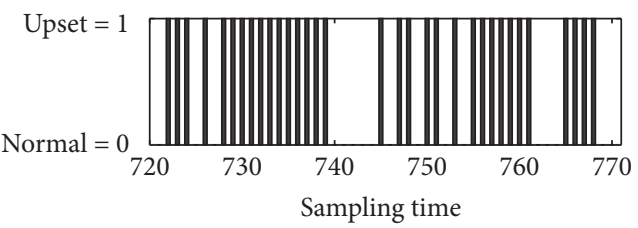

(f) Detailed upsets in Channel 3

FIGURE 4: Digital upsets distribution of each channel over three-CPE configuration generated by MDHMM model.

reality, the observation probability matrix is not a strict unit matrix, then this demonstrates that the digital upsets is not a strict first-order Markov process. Further, different observation probability matrices will make the digital upsets in each controller deviate from Markov process characteristics.

When regarding digital upsets generated by electromagnetic environment, the physical experiment analysis provided by literature [8] showed that the digital upsets process cannot be strictly described by Markov process. However, it can be appropriately described as a first-order Markov process with low confidence level of hypothesis testing. It can be seen that the digital upset signals contain the Markov characteristics of hidden state transition to a certain extent. Therefore, the transition characteristics of the digital upsets can be counted and used in the initial state transition probability matrix of MDHMM by the following:

$$
\begin{aligned}
& a_{i j}^{f}=\frac{\mathrm{P}\left\{S_{j}^{f}(k) \mid S_{i}^{f}(k-1)\right\}}{\mathrm{P}\left\{S_{i}^{f}(k)\right\}} \\
& a_{i j}=\frac{1}{F} \sum_{f=1}^{F} a_{i j}^{f}
\end{aligned}
$$

where $i, j \in\{1,2, \ldots, N\}, f \in\{1,2, \ldots, F\}$ and $k$ is the sampling instant. Then the initial observation probability matrix $\boldsymbol{B}_{h_{0}}^{f}$ is randomly chosen and all of its elements take value of 0.5 . After choosing the initial value of MDHMM, the traditional Baum-Welch algorithm iterates until achieving the suitable convergence. In order to meet the application accuracy requirement, the convergence conditions of BaumWelch algorithm are shown as follows: the difference between two iterations of the normalized likelihood probability, the $\|\cdot\|_{2}$ of the state transition matrix $A_{h}$ and the observation matrix $\boldsymbol{B}_{h}$, is less than $1 \times 10^{-6}$.

It is worth mentioning that due to the inherent difficulty of MDHMM parameters training, although the model parameters training method presented in this paper cannot guarantee the global optimal result, it can still quickly converge to the local optimal solution under the given accuracy requirements, taking into account the accuracy and the efficiency of the algorithm. The paper considers the airworthiness requirements; then the initial conditions must be in normal mode during taking-off phase.

\section{Theoretical Performance Analysis of Markov Switched Flight Control Systems under Correlated Digital Upsets}

3.1. General Theoretical Performance Analysis of Markov Switched Flight Control Systems. As mentioned above, the literatures [1-8] carried out theoretical and simulation analyses of the switched flight control system performance under stochastic Markov digital random interferences. In this section, the paper, first, introduces the fundamental relevant theorems about the bounded linear operator [22].

Let $C$ be an $n$-dimensional complex Euclidean space, and $M\left(\mathrm{C}^{m}, \mathrm{C}^{n}\right)$ be a normed linear space composed of all $n \times m \mathrm{~d}$ imensional matrices and be abbreviated as $M\left(C^{n}\right)$ when $n=m$.

Definition 1. Column stacking operator, $\widehat{\varphi}$, has the following operation for the matrix $H_{i} \in M\left(\mathrm{C}^{m}, \mathrm{C}^{n}\right), i=1,2, \ldots, N$ :

$$
\widehat{\varphi}\left(\boldsymbol{H}_{1}, \boldsymbol{H}_{2}, \ldots, \boldsymbol{H}_{N}\right):=\left[\begin{array}{c}
\boldsymbol{H}_{1} \\
\boldsymbol{H}_{2} \\
\vdots \\
\boldsymbol{H}_{N}
\end{array}\right] \in M\left(\mathrm{C}^{m}, \mathrm{C}^{N n}\right),
$$


Definition 2. The inverse column stacking operator, $\widehat{\varphi}_{j}^{-1}$, has the following operation for the matrix $H_{j} \in M\left(C^{m}, C^{n}\right), j=1$, $2, \ldots, N$ :

$$
\widehat{\varphi}_{j}^{-1}\left(\left[\begin{array}{c}
\boldsymbol{H}_{1} \\
\boldsymbol{H}_{2} \\
\vdots \\
\boldsymbol{H}_{N}
\end{array}\right]\right)=\boldsymbol{H}_{j} \in M\left(\mathrm{C}^{m}, \mathrm{C}^{n}\right) \text {, }
$$

Definition 3. For any bounded initial state $x_{0}$ of any $n$ dimensional switched linear system, if there is a non-negative real number $\alpha$ which makes $E\left\{\|x(k)\|^{2}\right\} \longrightarrow \alpha$ when time $k \longrightarrow \infty$, the switched linear system is mean-square stable.

The switched linear system (1a) and (1b) has the above mean-square stable characteristics.

Lemma 4 (see[5]). Markovian switched linear system (2a) and $(2 b)$ is mean-square stable if and only if $\boldsymbol{r}_{\sigma}\left(\mathscr{A}_{2}\right)<1$, where

$$
\begin{aligned}
& \mathscr{A}_{2} \\
& \quad=\operatorname{diag}\left(A_{e, 0}^{T} \otimes A_{e, 0}^{T}, A_{e, 1}^{T} \otimes A_{e, 1}^{T}, \ldots, A_{e, l-1}^{T} \otimes A_{e, l-1}^{T}\right) \\
& \quad \cdot\left(\Pi_{v} \otimes I_{n^{2}}\right)
\end{aligned}
$$

where $\Pi_{\boldsymbol{v}}$ is state transition matrix of Markovian switched law, $\boldsymbol{v}(k) . r_{\sigma}$ is the spectral radius of the matrix.

In this paper, the above lemma is applied to estimate the system stability. When the closed-loop flight control system is stable under digital interferences, the index $\boldsymbol{J}_{w, e}$ can be estimated by the following lemma and theorems derived from Theorem 3 in [8] to evaluate the control system performance degradation.

Lemma 5 (see [8]). For the mean-square stable Markovian switched linear system (2a) and (2b), if the switching law, $\boldsymbol{v}(k)$, is aperiodic and ergodic, the initial state is $x_{0}=0$, and the white Gaussian noise input $\omega(t)$ is independent of the switching law, $\boldsymbol{v}(k)$, then

$$
\begin{aligned}
\overline{\boldsymbol{C}}_{i} & =\boldsymbol{C}_{i}^{\mathrm{T}} \cdot \boldsymbol{C}_{i} \\
V_{j} & =\sum_{i=0}^{l-1}\left[\Pi_{\boldsymbol{v}}\right]_{i j} \boldsymbol{B}_{i} \boldsymbol{B}_{i}^{T}[p]_{i} \\
\overline{\mathbf{Q}}_{i} & =\widehat{\varphi}^{-1}\left(\left(\boldsymbol{I}_{l n^{2}}-\mathscr{A}_{2}^{\mathrm{T}}\right)^{-1} \cdot \varphi\left(\boldsymbol{V}_{0}, \boldsymbol{V}_{1}, \ldots, \boldsymbol{V}_{l-1}\right)\right) \\
J_{w, e} & =\sum_{i=0}^{l-1} \operatorname{tr}\left(\overline{\boldsymbol{C}}_{i} \cdot \overline{\mathbf{Q}}_{i}\right)
\end{aligned}
$$

where $l=2, \Pi_{v}$ is state transition matrix of Markovian switching law $\boldsymbol{v}(k)$, and $\left[\Pi_{\boldsymbol{v}}\right]_{i j}$ represents the ij-th element of the matrix $\Pi$.

It is noted that the switching law of the switched linear system is the first-order Markov chain for Lemmas 4 and 5.
However, the observation processes of HMM and MDHMM, in general, do not have Markovian property. Therefore, those two lemmas cannot be directly applied to the stability and performance analysis.

The switched flight control system driven by HMM digital random interferences is described as

$$
\begin{gathered}
\boldsymbol{x}(k+1)=\boldsymbol{A}_{o_{k}} \boldsymbol{x}(k)+\boldsymbol{B}_{o_{k}} \boldsymbol{w}(k) \\
\boldsymbol{y}(k)=\boldsymbol{C}_{o_{k}} \boldsymbol{x}(k)
\end{gathered}
$$

where the switching law $o_{k}$ is generally not a Markov chain. However, the joint process among Markov and non-Markov processes is a Markov chain [8]. Then the switched flight control system driven by the joint process, $\rho(k)$, is denoted as

$$
\begin{gathered}
\boldsymbol{x}(k+1)=\boldsymbol{A}_{\rho(k)} \boldsymbol{x}(k)+\boldsymbol{B}_{\rho(k)} \boldsymbol{w}(k) \\
\boldsymbol{y}(k)=\boldsymbol{C}_{\rho(k)} \boldsymbol{x}(k)
\end{gathered}
$$

The switching law $\rho(k)$ of the system is a first-order Markov chain. In order to use system (11a) and (11b) to analyze system (10a) and (10b), it is necessary to ensure that the state space model are identical at each time. Then we have to define the switched systems equivalence.

Definition 6. For switched systems (10a), (10b), (11a), and (11b), if for any time $k$,

$$
\begin{aligned}
& A_{o_{k}}=A_{\rho(k)}, \\
& \boldsymbol{B}_{o_{k}}=\boldsymbol{B}_{\rho(k)}, \\
& \boldsymbol{C}_{o_{k}}=\boldsymbol{C}_{\rho(k)},
\end{aligned}
$$

then those two switched systems are called equivalent.

When switched systems (10a), (10b), (11a), and (11b) are equivalent, one can use the existing Markovian switched linear system theory to estimate the flight system (11a) and (11b) performance degradation driven by HMM digital interferences.

3.2. Theoretical Performance Analysis of Markovian Switched Flight Control Systems under Correlated Digital Upsets Modeled by MDHMM. This section considers the theoretical performance degradation model for Boeing 747 flight control system based on the structures shown in Figures 1-3. The existing traditional methods for multi-channel flight control system performance estimation have two prerequisites. First, the digital random interference processes for each channel are independent of each other. Second, the digital random interference process for each channel has Markov properties. However, in the MDHMM model, the hidden "electromagnetic state" is always characterized by a first-order Markov chain. In reality, the digital random interferences of each channel are related to the electromagnetic states and described by the HMMs which do not have the Markov properties. In addition, the interference process among channels in the MDHMM model is conditionally independent 
of hidden state variables instead of mutually independent. Compared to the traditional mutually independent Markov interference processes, MDHMM interference processes are much more complex. However, it can better characterize the relationship between electromagnetic environments and correlated digital upsets injected into the flight control system. Therefore, it is necessary to develop a new method to analyze the performance degradation of flight control systems under correlated digital upsets by using MDHMM.

Then reconsider the inherent logical relationship among state variables and stochastic digital upsets modeled by MDHMM. The random hidden electromagnetic state variable at time $k$ is only related to that at time $k$ - 1 , regardless of states at all previous moments for Markov processes. Meanwhile, the random digital interference upset of each channel is only related to the hidden electromagnetic state variable at time $k$. Therefore, the joint random process among the random electromagnetic state and the digital interference upsets of each channel have Markov properties as well which is elaborated in Theorem 7.

Theorem 7. For F-dimensional MDHMM model $\lambda=$ $\left(\boldsymbol{S}, \boldsymbol{V}, \boldsymbol{A}_{h}, \boldsymbol{B}_{h}^{f}, \boldsymbol{\pi}\right)$, the state transition matrix is $\boldsymbol{A}_{h}=\left[a_{i j}\right]$, the observation probability matrix is $\boldsymbol{B}_{h}^{f}=\left[b_{j}^{f}(l)\right], i, j \in\{1,2$, $\ldots, N\}, f \in\{1,2, \ldots, F\}, l \in\left\{1,2, \ldots, M_{f}\right\}$. As shown in Figure 5, a hidden state variable is $\boldsymbol{q}(k)$ at time $k$, and the observation state vector is $\boldsymbol{o}(k)=\left(o_{k}^{1}, o_{k}^{2}, \ldots, o_{k}^{F}\right)$. Then the joint process

$$
\overline{\boldsymbol{u}}(k):=(\boldsymbol{q}(k), \boldsymbol{o}(k))
$$

is a first-order Markov chain with $N \times M^{F}$ states, and the corresponding state transition probability is

$$
\begin{aligned}
v_{\left(i, g_{1}, g_{2}, \ldots, g_{F}\right)\left(j, l_{1}, l_{2}, \ldots, l_{F}\right)}= & a_{i j} \cdot b_{j}^{1}\left(l_{1}\right) \cdot b_{j}^{2}\left(l_{2}\right) \cdot \ldots \\
& \cdot b_{j}^{F}\left(l_{F}\right)
\end{aligned}
$$

Proof. Let the state of the joint process at time $k-1$ is

$$
\begin{aligned}
\overline{\boldsymbol{u}}(k-1) & =\left(\boldsymbol{q}(k-1)=i, \boldsymbol{o}(k)=\left(g_{1}, g_{2}, \ldots, g_{F}\right)\right) \\
& =\left(i, g_{1}, g_{2}, \ldots, g_{F}\right)
\end{aligned}
$$

and let the state of the joint process at time $k$ is

$$
\begin{aligned}
\overline{\boldsymbol{u}}(k) & =\left(\boldsymbol{q}(k)=j, \boldsymbol{o}(k)=\left(l_{1}, l_{2}, \ldots, l_{F}\right)\right) \\
& =\left(j, l_{1}, l_{2}, \ldots, l_{F}\right)
\end{aligned}
$$

According to the definition of MDHMM, the following conditional probability are

$$
\begin{aligned}
P & \left\{\overline{\boldsymbol{u}}(k)=\left(j, l_{1}, l_{2}, \ldots, l_{F}\right) \mid \overline{\boldsymbol{u}}(k-1)\right. \\
& \left.=\left(i, g_{1}, g_{2}, \ldots, g_{F}\right)\right\}=P\{\boldsymbol{q}(k)=j, \boldsymbol{o}(k) \\
& =\left(l_{1}, l_{2}, \ldots, l_{F}\right) \mid \boldsymbol{q}(k-1)=i, \boldsymbol{o}(k-1)
\end{aligned}
$$

$$
\begin{aligned}
& \left.=g_{1}, g_{2}, \ldots, g_{F}\right\}=P\{\boldsymbol{q}(k)=j, \boldsymbol{o}(k) \\
& \left.=\left(l_{1}, l_{2}, \ldots, l_{F}\right) \mid \boldsymbol{q}(k-1)=i\right\}=P\{\boldsymbol{q}(k) \\
& =j \mid \boldsymbol{q}(k-1)=i\} \cdot P\left\{\boldsymbol{o}(k)=\left(l_{1}, l_{2}, \ldots, l_{F}\right) \mid \boldsymbol{q}(k)\right. \\
& =j\}=a_{i j} \cdot b_{j}^{1}\left(l_{1}\right) \cdot b_{j}^{2}\left(l_{2}\right) \cdot \ldots \cdot b_{j}^{F}\left(l_{F}\right)
\end{aligned}
$$
chain.

Therefore, the joint process $\overline{\boldsymbol{u}}(k)$ is a first-order Markov

Theorem 8. o(k) are digital homogeneous Markov upset processes injected into N CPEs distributed control system on a state space $\{0,1\}^{N}$ described by $N$ dimensional MDHMM model. Define the hidden state of the MDHMM model as $\boldsymbol{q}(k)$, the stochastic process $v(k):=\phi\left(o_{i}(k)\right)$, and the joint process $\widehat{y}(k):=\left(o_{1}(k), \cdots, o_{N}(k)\right)$, and then the joint process $\rho(k)$

$$
\boldsymbol{\rho}(k):=(\boldsymbol{q}(k), \widehat{\boldsymbol{y}}(k), \boldsymbol{v}(k))
$$

is the homogeneous Markov chain and the corresponding state space can be reduced into a proper subset of $\{0,1\}^{N+1}$.

Proof. According to Theorem 7, it is known that

$$
\overline{\boldsymbol{u}}(k)=(\boldsymbol{q}(k), \boldsymbol{o}(k))
$$

Therefore,

$$
\boldsymbol{\rho}(k)=(\overline{\boldsymbol{u}}(k), \boldsymbol{v}(k))
$$

Then

$$
\begin{aligned}
\mathrm{P} & \{\boldsymbol{\rho}(k) \mid \boldsymbol{\rho}(k-1)\} \\
& =\mathrm{P}\{\overline{\boldsymbol{u}}(k), \boldsymbol{v}(k) \mid \overline{\boldsymbol{u}}(k-1), \boldsymbol{v}(k-1)\} \\
& =\mathrm{P}\{\overline{\boldsymbol{u}}(k) \mid \overline{\boldsymbol{u}}(k-1)\}
\end{aligned}
$$

Therefore, based on Theorem 7, the joint process $\rho(k)$ is a homogeneous Markov chain. Then the joint process $\rho(k)$ can be used to drive the switched flight control system (2a) and (2b), and Lemma 5 can analyze the theoretical performance error of system (2a) and (2b).

\section{An Example of Flight Control System Performance Analysis under Correlated Upsets Modeled by MDHMM}

Consider the dual-channel flight control system subject to digital stochastic upsets described by MDHMM, the corresponding hidden environmental and observation matrices are shown as follows:

$$
\begin{aligned}
& \boldsymbol{A}_{h}=\left[\begin{array}{ll}
0.9991 & 0.0009 \\
0.0322 & 0.9678
\end{array}\right] \\
& \boldsymbol{B}_{h}^{o_{1}}=\left[\begin{array}{ll}
0.9993 & 0.0007 \\
0.1779 & 0.8221
\end{array}\right] \\
& \boldsymbol{B}_{h}^{o_{2}}=\left[\begin{array}{ll}
0.9994 & 0.0006 \\
0.1777 & 0.8223
\end{array}\right]
\end{aligned}
$$




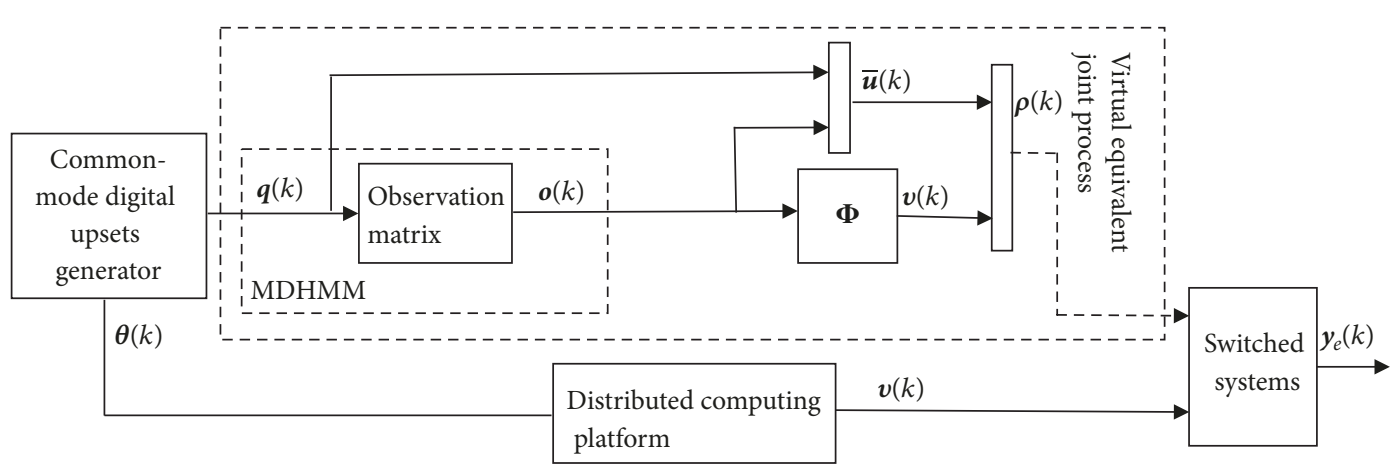

FIGURE 5: Joint process conceptual schematic over the distributed configuration under digital upsets modeled by MDHMM.

The joint process state table for $2 \mathrm{CPEs}$ structure for switched flight control system subject to digital upsets as shown in Figure 1 is listed in Table 1.

The joint process $\rho(k)$ is a first-order Markov chain with eight states. The eight state transition matrices can be obtained by Theorem 7 , and $\mathscr{A}_{2}$ can be calculated by

$$
\begin{aligned}
\mathscr{A}_{2}= & \operatorname{diag}\left(\boldsymbol{A}_{\rho_{1}}^{T} \otimes A_{\rho_{1}}^{T}, A_{\rho_{2}}^{T} \otimes A_{\rho_{2}}^{T}, \ldots, A_{\rho_{8}}^{T} \otimes A_{\rho_{8}}^{T}\right) \\
& \cdot\left(\Pi_{\rho} \otimes I_{54^{2}}\right)
\end{aligned}
$$

The switching law $\rho(k)$ of the switched flight control system is equivalent to the actual system switching signal $\boldsymbol{v}(k)$, then

$$
\begin{aligned}
& \mathscr{A}_{2}=\operatorname{diag}\left(\boldsymbol{A}_{0}^{T} \otimes \boldsymbol{A}_{0}^{T}, \boldsymbol{A}_{0}^{T} \otimes \boldsymbol{A}_{0}^{T}, \boldsymbol{A}_{0}^{T} \otimes \boldsymbol{A}_{0}^{T}, \boldsymbol{A}_{1}^{T}\right. \\
& \left.\otimes \boldsymbol{A}_{1}^{T}, \boldsymbol{A}_{0}^{T} \otimes \boldsymbol{A}_{0}^{T}, \boldsymbol{A}_{0}^{T} \otimes \boldsymbol{A}_{0}^{T}, \boldsymbol{A}_{0}^{T} \otimes \boldsymbol{A}_{0}^{T}, \boldsymbol{A}_{1}^{T} \otimes \boldsymbol{A}_{1}^{T}\right)\left(\Pi_{\rho}\right. \\
& \left.\quad \otimes \boldsymbol{I}_{54^{2}}\right)
\end{aligned}
$$

The general radius of the matrix $\mathscr{A}_{2}, r_{\sigma}\left(\mathscr{A}_{2}\right)$, is $0.9734<1$, therefore, the switched system $(2 \mathrm{a})$ and $(2 \mathrm{~b})$ is mean-square stable. The system performance degradation is $9.3575 \times 10^{-7}$, an acceptable performance degradation.

In order to verify the accuracy of the theoretical analysis, simulation verification by using Monte Carlo method was carried out by MATLAB. First, the simulated digital upset processes by the HMM model through training are generated. Second, the error model of the flight control system (11a) and (11b) is driven by these digital upsets. Third, the mean of the steady-state part of the average system output response after 200,000 Monte Carlo runs are calculated. Finally, the corresponding performance error output of the switched system is 9.3575 e-7, which meets the theoretical analysis result.

\section{Simulation Verifications for Flight Control System Performance Analysis under Correlated Upsets Modeled by MDHMM}

Four redundant architectures with $1 \mathrm{CPE}, 2 \mathrm{CPEs}, 3 \mathrm{CPEs}$, and $4 \mathrm{CPEs}$ are established under a certain environment by using the independent HMM model and the correlated MDHMM model, respectively. The digital upset process was simulated and fed into the switched flight control system. Then the comparisons of the average error output responses among MDHMM model and the corresponding independent HMM model for each architecture are shown in Figure 6 after the total 200,000 Monte Carlo runs.

In Figure 6, four subgraphs use semilogarithmic coordinate systems, where the vertical coordinate of performance errors is in logarithmic scale. As shown in Figure 6, the degradations of the two models in Figure 6(a) are basically the same. The MDHMM model becomes the HMM model in the 1 CPE structure. Those two are essentially the same model with the same characteristics. As shown in Figures 6(b)-6(d), it is easy to observe that the performance errors by using the independent HMM model are several orders of magnitude better than that by using the MDHMM model. It is known that in the independent HMM upsets model, the digital upsets in different CPEs are rarely happened simultaneously. Then, at least one CPE can be guaranteed to operate normally at each sampling time. However, the HIRF environment always generates common-mode upsets and affects all CPEs at the same time, which might cause the overall system fail intermittently. However, current literatures demonstrated that the common-mode characteristics of digital upsets can be reflected more accurately by MDHMM model than others. Therefore, it is reasonable that the performance of the overall system subject to upsets modeled by MDHMM is worse than that modeled by HMM.

Based on the established MDHMM model of digital upset processes for different redundant structures, Lemma 5 and Theorem 7 are used to predict the performance degradation of the flight control system under simulated different field strength environments. For the simulation analyses, the generated simulated digital upset signals generated by MDHMM models are used to drive the switched flight control system under simulated electromagnetic environments whose field intensity ranges from $100 \mathrm{~V} / \mathrm{m}$ to $180 \mathrm{~V} / \mathrm{m}$ increased by every $10 \mathrm{~V} / \mathrm{m}$ at frequency $100 \mathrm{MHz}$. Each experiment carries out 200,000 Monte Carlo runs. Then the comparisons between the theoretical prediction and simulation estimation results under different redundant structures and environments are conducted and shown in Table 2. Table 2 demonstrates that theoretical estimations of the performance degradation match the simulation results efficiently for four kinds of redundant structures under different strength environments. 
Table 1: State Table for 2 CPEs Redundant Structure of Flight Control System Subject to Environments.

\begin{tabular}{lcccc}
\hline $\boldsymbol{q}(k)$ & $\boldsymbol{y}_{2}(k)$ & $\boldsymbol{v}(k)=\phi\left(\boldsymbol{y}_{i}(k)\right)$ & $\boldsymbol{\rho}(k):=(\boldsymbol{q}(k), \hat{\boldsymbol{y}}(k), \boldsymbol{v}(k))$ \\
\hline 0 & 0 & 0 & 0 & $(000,0)$ \\
0 & 0 & 1 & 0 & $(001,0)$ \\
0 & 1 & 0 & 0 & $(010,0)$ \\
0 & 1 & 1 & 1 & $(011,1)$ \\
1 & 0 & 0 & 0 & $(100,0)$ \\
1 & 0 & 1 & 0 & $(101,0)$ \\
1 & 1 & 0 & 0 & $(110,0)$ \\
1 & 1 & 1 & 1 & $(111,1)$ \\
\hline
\end{tabular}

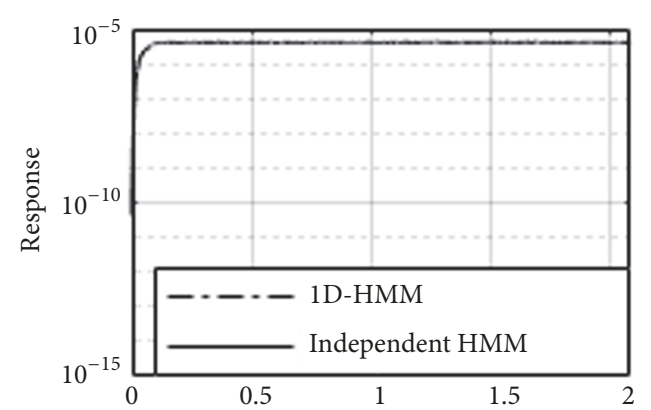

(a) Sampling Time $\times 10^{4}$. One CPE

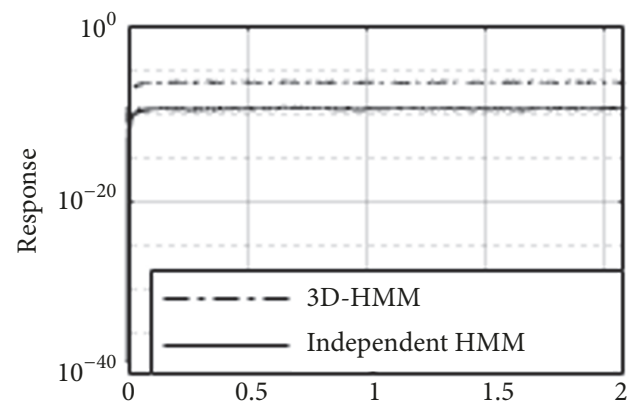

(c) Sampling Time $\times 10^{4}$. Three CPEs Redundancy

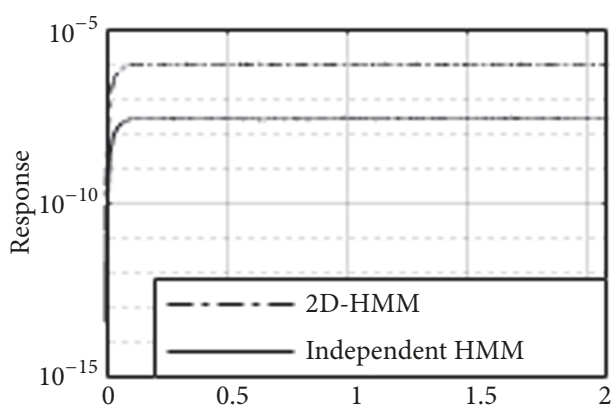

(b) Sampling Time $\times 10^{4}$. Two CPEs Redundancy

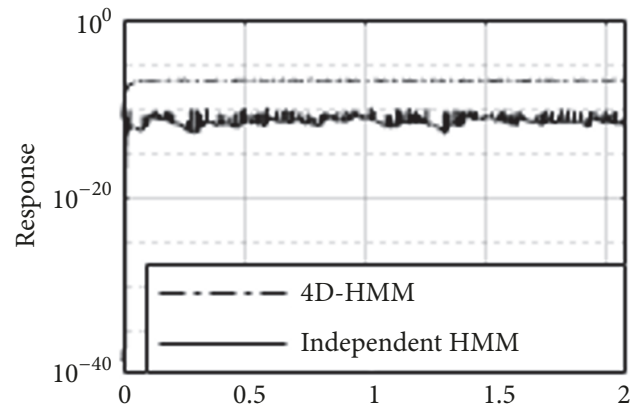

(d) Sampling Time $\times 10^{4}$. Four CPEs Redundancy

FIGURE 6: Comparison of the performance degradation response between the MDHMM model and the corresponding independent HMM model for four redundant structures.

As shown in Figure 7, it is easy to find that the flight performance error increases as the field strength of electromagnetic environment increases and is getting worse. The redundant fault-tolerant control structure can reduce the performance error, however, it is hard to draw a conclusion that the more the better straightforwardly. For example, performance error for $2 \mathrm{CPEs}$ structure is less than that for $1 \mathrm{CPE}$ structure and has an obviously greater performance improvement. However, the improvement between $3 \mathrm{CPEs}$ and $4 \mathrm{CPEs}$ structures for the performance error is less significant. It is known that the common-mode interferences caused by the electromagnetic environment will affect all CPEs at the same time. Meanwhile, the more redundant units, the more difficult the design and the higher maintenance cost of the flight control system, therefore, it is better to balance the improvement of flight performance and the redundancy units.

Figure 8 shows the performance error response comparison among four kinds of redundant structures under a certain simulated electromagnetic environment by using MDHMM. As shown in Figure 8, the mean-square stable characteristics are obvious for four different fault-tolerant structures under digital stochastic upsets. The performance error response becomes stable quickly for each structure. It is obvious that the magnitudes of the performance errors are very small which satisfy flight safety requirements. This is consistent with the results shown in Figure 7.

\section{Conclusions}

The paper discussed the common-source digital upsets modeled by MDHMM and revealed the relationship between the environments and observed digital upsets. It is found that this is a more accurate model used for characterizing correlated upsets compared to the existing HMM approach. Furthermore, the theoretical performance degradation analysis method was developed under common-source upsets 


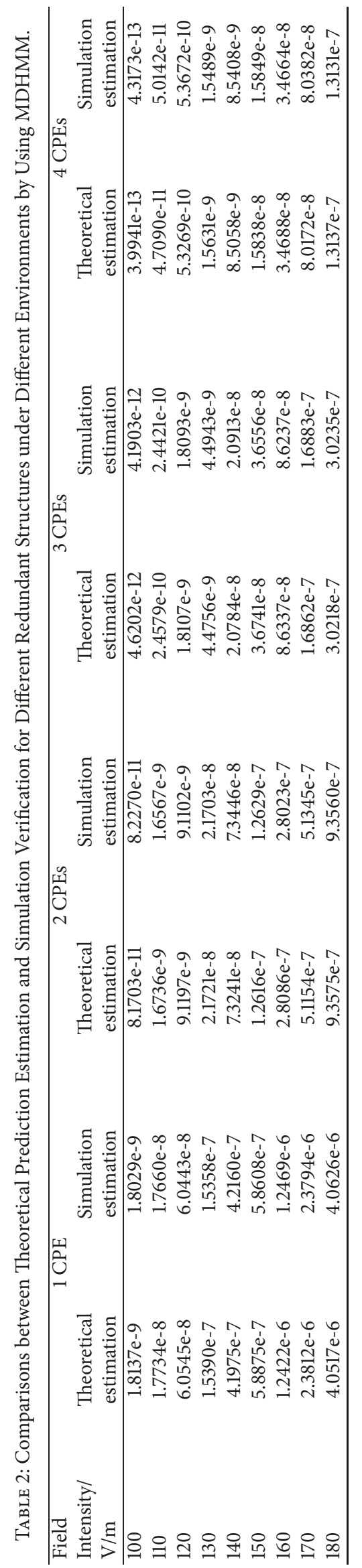




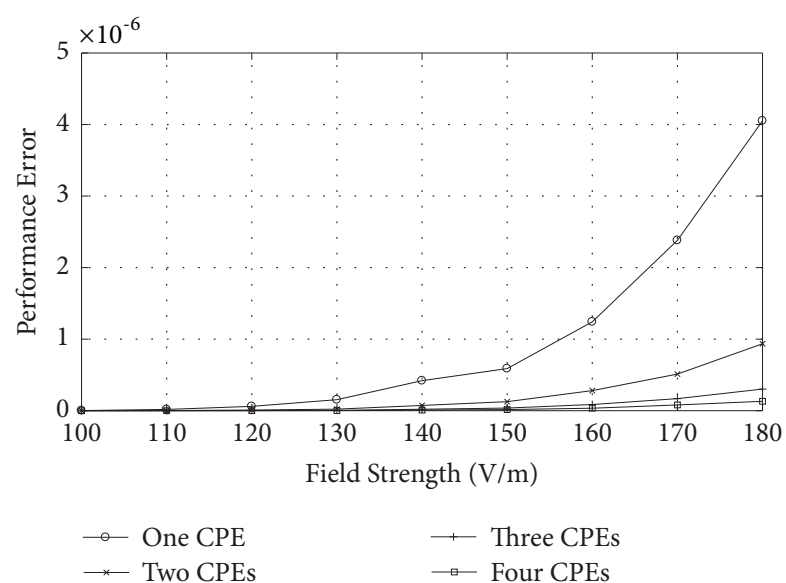

FIGURE 7: Simulation estimation comparisons of performance error for different redundant structures under different environments modeled by MDHMM.

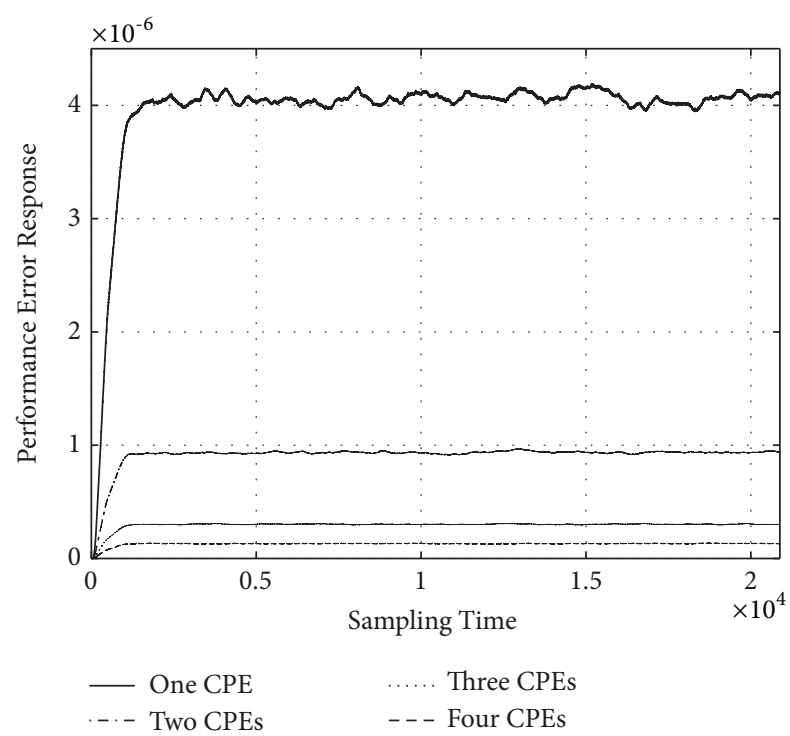

FIGURE 8: Simulation estimation of the performance error response for different redundant structures subject to common-source digital upsets under a certain simulated electromagnetic field.

modeling by the MDHMM. This theoretical analysis method integrated with the system dynamics aircraft undertakes, the redundant structure the flight design characterizes, and the MDHMM model the disturbances satisfy. Then simulation verifications for the performance degradation of a distributed redundant flight control system were conducted by Monte Carlo methods when the system was subject to correlated common-source upsets. Finally, the comparisons among the theoretical analyses and the simulation verifications of the control system demonstrated the accuracy of the theoretical method which can benefit flight control systems redundancy designs. Theoretical stability analyses of the mentioned structures were also analyzed and demonstrated the mean-square stability property. The optimal design scheme will help to balance the performance the aircraft undertake, the weight of aircraft, and the maintenance costs in application.

\section{Data Availability}

The data used to support the findings of this study are included within the article.

\section{Conflicts of Interest}

The authors declare that there are no conflicts of interest regarding the publication of this paper.

\section{Acknowledgments}

This work was supported in part by the Key Project of Civil Aviation Joint Fund by the National Natural Science Foundation of China and CACC (Grant no. U1533201), the Fundamental Research Funds for the Central UniversitiesCivil Aviation University of China (3122017003), Natural Sciences and Engineering Research Council of Canada, and the Scientific Research Foundation for the Returned Overseas Chinese Scholars, State Education Ministry. Tianjin Key Laboratory of Civil Aircraft Airworthiness and Maintenance in CAUC (104003020106).

\section{References}

[1] W. S. Gray and O. R. González, "EMI models for controller analysis and synthesis," in Proceedings of the Proc. 17th Digital Avionics Systems Conf, Seattle, 1998.

[2] W. Gray, O. Gonzalez, and M. Dogan, "Stochastic perturbation models of electromagnetic disturbances in closed-loop computer controlled flight systems," in Proceedings of the Gateway to the New Millennium. 18th Digital Avionics Systems Conference. Proceedings, pp. 10.C.4-1-10.C.4-8, St Louis, MO, USA.

[3] W. Gray, O. Gonzalez, and S. Patilkulkarni, "Stability of digital control systems subject to jump linear random perturbations," in Proceedings of the 39th IEEE Conference on Decision and Control, pp. 1154-1159, Sydney, NSW, Australia.

[4] W. S. Gray, O. R. González, and M. Doǧan, "Stability analysis of digital linear flight controllers subject to electromagnetic disturbances," IEEE Transactions on Aerospace and Electronic Systems, vol. 36, no. 4, pp. 1204-1218, 2000.

[5] H. Zhang, W. S. Gray, and O. R. González, "Performance analysis of digital flight control systems with rollback error recovery subject to simulated neutron-induced upsets," IEEE Transactions on Control Systems Technology, vol. 16, no. 1, pp. 46-59, 2008.

[6] H. Zhang, W. S. Gray, O. R. Gonzalez, and A. V. Lakdawala, "Output Tracking Performance of A Recoverable Digital Flight Control System in Neutron Environments," IEEE Transactions on Aerospace and Electronic Systems, vol. 45, no. 1, pp. 321-335, 2009.

[7] C. Belcastro, "Closed-loop HIRF experiments performed on a fault tolerant flight control computer," in Proceedings of the 16th DASC. AIAA/IEEE Digital Avionics Systems Conference. Reflections to the Future. Proceedings, pp. 4.1-40-54, Irvine, CA, USA.

[8] R. Wang, W. Steven Gray, O. R. Gonzalez, and J. R. ChavezFuentes, "Tracking performance of distributed recoverable 
flight control systems subject to high intensity radiated fields," IEEE Transactions on Aerospace and Electronic Systems, vol. 49, no. 1, pp. 521-542, 2013.

[9] R. Wang, W. S. Gray, and O. R. Gonzalez, "Experimental validation of a performance model for a distributed recoverable Boeing 747 flight control system subject to digital upsets," in Proceedings of the 2011 IEEE 43rd Southeastern Symposium on System Theory (SSST 2011), pp. 11-17, Auburn, AL, USA, March 2011.

[10] W. S. Gray, R. Wang, O. R. González, and J. R. Chávez-Fuentes, "Tracking performance analysis of a distributed recoverable boeing 747 flight control system subject to digital upsets," in Proceedings of the 2010 American Control Conference, ACC 2010, pp. 548-554, USA, July 2010.

[11] W. S. Gray, R. Wang, and O. R. Gonzalez, "A performance model for a distributed flight control system subject to random upsets," in Proceedings of the 2008 IEEE International Conference on Control Applications (CCA) part of the IEEE Multi-Conference on Systems and Control, pp. 918-923, San Antonio, TX, USA, September 2008.

[12] Y. Li and R. Wang, "The flight control system performance analysis based on hidden Markov switched linear systems," in Proceedings of the 2016 35th Chinese Control Conference (CCC), pp. 2063-2067, Chengdu, China, July 2016.

[13] R. Wang, Y. Li, H. Sun, and Z. Chen, "Performance analysis of switched flight control systems based on hidden Markov model," Journal of Electronics \& Information Technology, vol. 39, no. 4, pp. 989-996, 2017.

[14] R. Wang, H. Sun, Y. Li, and Y. Zhang, "The common-source digital upsets analysis by MDHMM for control system performance," in Proceedings of the 2017 IEEE International Conference on Systems, Man and Cybernetics (SMC), pp. 2093-2098, Banff, AB, October 2017.

[15] J. Baumgartner, A. G. Flesia, J. Gimenez, and J. Pucheta, "A new image segmentation framework based on two-dimensional hidden Markov models," Integrated Computer-Aided Engineering, vol. 23, no. 1, pp. 1-13, 2015.

[16] N. Malešević, D. Marković, G. Kanitz, M. Controzzi, C. Cipriani, and C. Antfolk, "Vector Autoregressive Hierarchical Hidden Markov Models for Extracting Finger Movements Using Multichannel Surface EMG Signals," Complexity, vol. 2018, 2018.

[17] J. Bobulski, "Multimodal face recognition method with twodimensional hidden Markov model," Bulletin of the Polish Academy of Sciences-Technical Sciences, vol. 65, no. 1, pp. 121128, 2017.

[18] P. Jiao, K. Xu, S. Yue, X. Wei, and L. Sun, "A decentralized partially observable Markov decision model with action duration for goal recognition in real time strategy games," Discrete Dynamics in Nature and Society, Art. ID 4580206, 15 pages, 2017.

[19] A. Tenyakov, R. Mamon, and M. Davison, "Modelling highfrequency FX rate dynamics: A zero-delay multi-dimensional HMM-based approach," Knowledge-Based Systems, vol. 101, pp. 142-155, 2016.

[20] J. M. Lee, S.-J. Kim, Y. Hwang, and C.-S. Song, "Diagnosis of mechanical fault signals using continuous hidden Markov model," Journal of Sound and Vibration, vol. 276, no. 3-5, pp. 1065-1080, 2004.

[21] L. R. Rabiner, "Tutorial on hidden Markov models and selected applications in speech recognition," Proceedings of the IEEE, vol. 77 , no. 2, pp. 257-286, 1989.

[22] O. L. V. Costa and M. D. Fragoso, "Stability results for discretetime linear systems with Markovian jumping parameters,"
Journal of Mathematical Analysis and Applications, vol. 179, no. 1, pp. 154-178, 1993. 


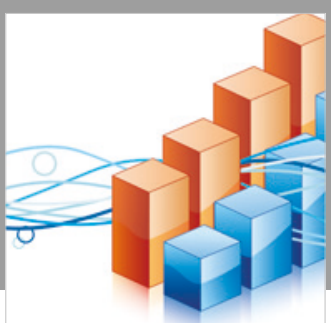

Advances in

Operations Research

\section{-n-m}
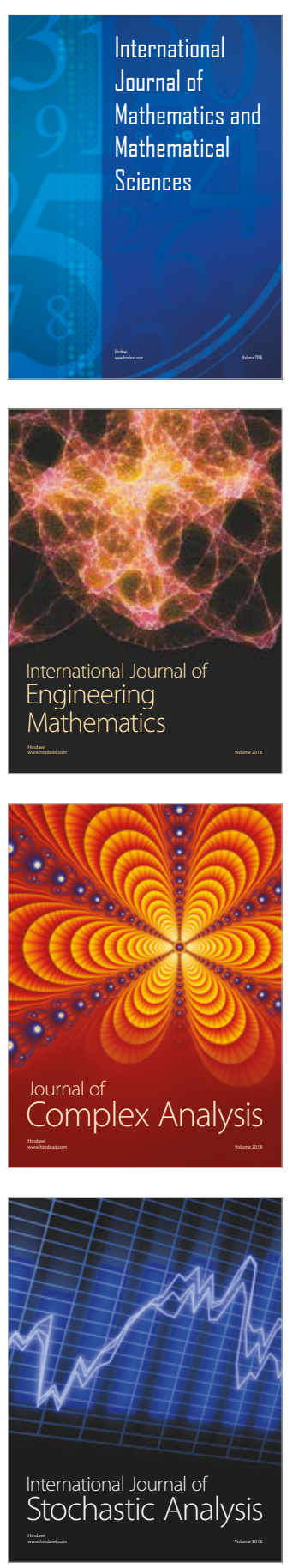
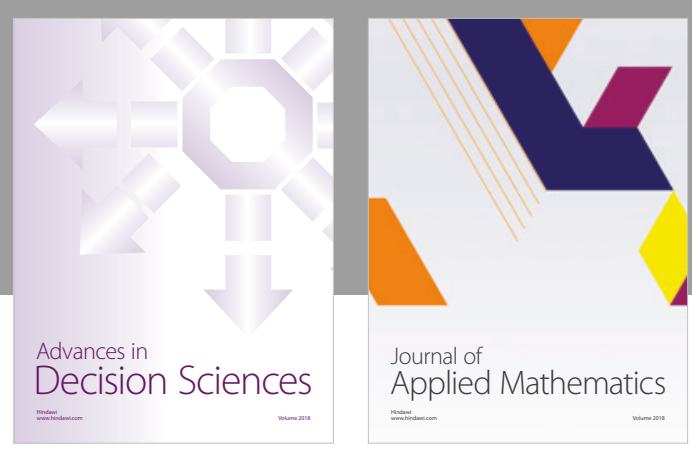

Journal of

Applied Mathematics
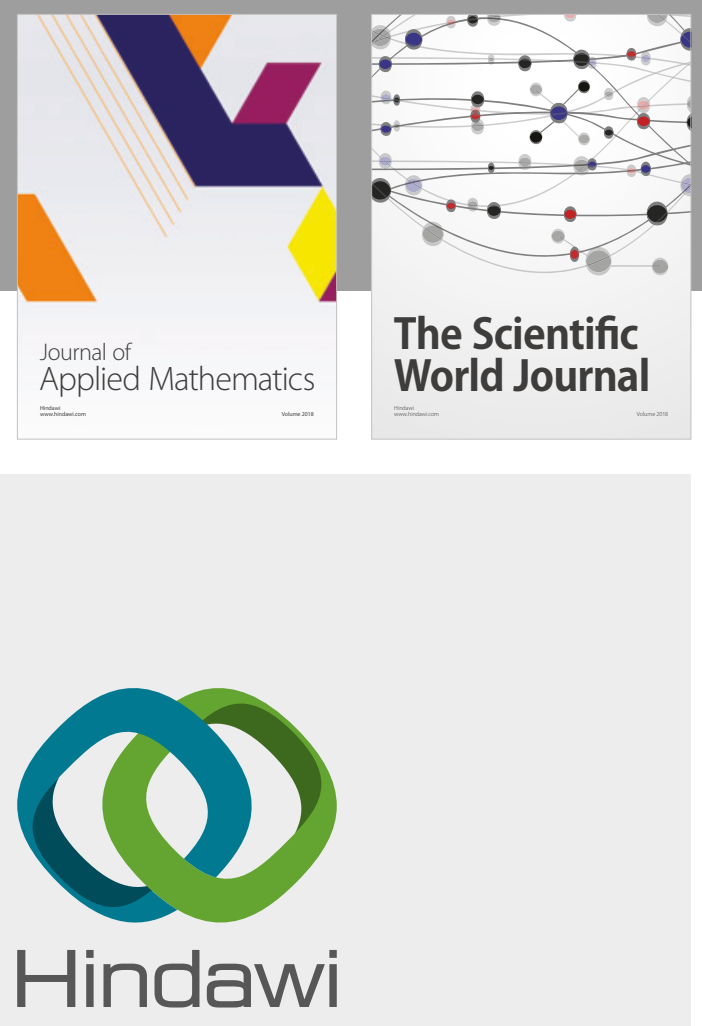

Submit your manuscripts at

www.hindawi.com

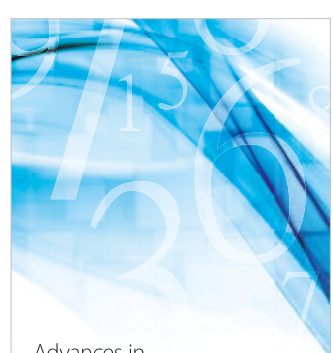

Advances in
Numerical Analysis
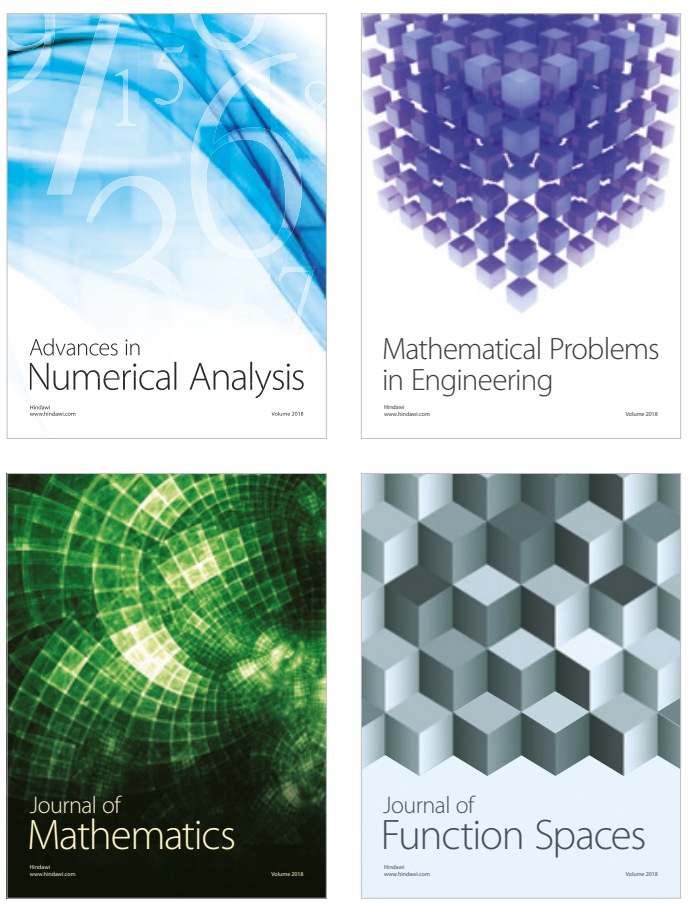

Mathematical Problems in Engineering

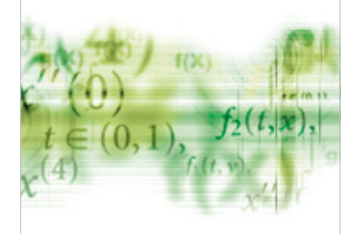

International Journal of

Differential Equations

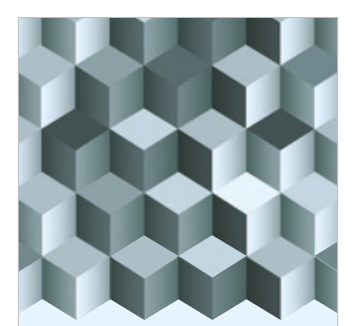

Journal of

Function Spaces
The Scientific

World Journal

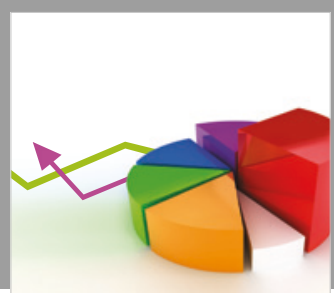

Journal of

Probability and Statistics
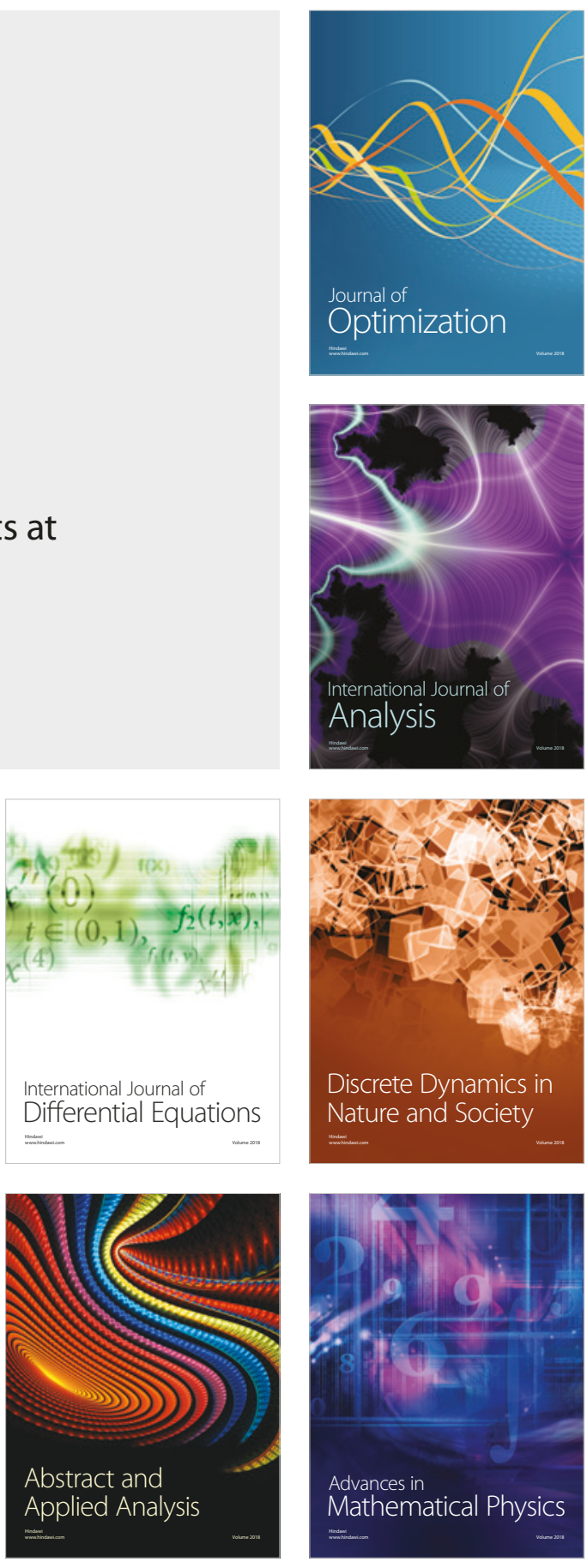